\title{
Extrahepatic Bile Duct Neuroendocrine Tumor
}

National Cancer Institute

\section{Source}

National Cancer Institute. Extrahepatic Bile Duct Neuroendocrine Tumor. NCI Thesaurus.

Code C96955.

A well differentiated, low or intermediate grade tumor with neuroendocrine

differentiation that arises from the extrahepatic bile ducts. 